\title{
Erratum
}

\section{Kaolinite-armoured polyurea microcapsules fabricated on Pickering emulsion: controllable encapsulation and release performance of a lipophilic compound - ERRATUM}

\author{
Cunjun Li, Minghao Wang, Zhaoliang Liu, Yanqi Xu, Chunhui Zhou and Linjiang Wang \\ https://doi:10.1180/clm.2021.15, Published by Cambridge \\ University Press, 11 June 2021.
}

Keywords: emulsion, interfacial polymerization, kaolinite, microcapsule, polyurea; erratum

The Publisher apologises to the Authors and to the readers for errors in the published article which were not corrected at proof stage. The corrections are as follows:

Vol. 56, No. 1, March 2021 issue

p. 46, abstract, line 1: instead of "applications", read "fields"

p. 46, right column, line 17: instead of "which were called later", read "and later referred to as"

p. 47, right column, line 12: instead of "Kln-embedded and armoured", read "Kln-embedded and -armoured".

p. 49, left column, line 12: instead of "Perkins Elmer", read "Perkin Elmer".

p. 50, Table 1, footnote b: instead of "..are the two marks of the same sample", read "are the same sample"; and footnote d: instead of "...are an approximations", read "are approximations"

p. 50, right column, lines 5-6: instead of "Such a phenomenon is ascribed to the habitation where Kln particles distributed in the previous $\mathrm{E}_{\mathrm{KIn}}$. That is," read "This difference between the internal and external surfaces is ascribed to the distribution of Kln particles in $\mathrm{E}_{\mathrm{Kln}}$, i.e."

p. 51, left column, line 35: instead of "range of 50-", read "range $50-"$

p. 53, left column, lines 31-32: instead of " $22 \%$ SR not given for $\mathrm{C}_{\mathrm{KP}-0.6 \mathrm{FPDI}}$ )." read "22\% SR remained for $\mathrm{C}_{\mathrm{KP}-0.6 \mathrm{SPDI}}$ after $45 \mathrm{~h}$ of release)."

p. 53 , right column, line 14: instead of "the $\mathrm{n}$ values are $>0.5$ ", read "no $n$ value is $>0.5$ "

\section{Reference}

Li C., Wang M., Liu Z., Xu Y., Zhou C. \& Wang L. Kaolinite-armoured polyurea microcapsules fabricated on Pickering emulsion: controllable encapsulation and release performance of a lipophilic compound. Clay Minerals. Published by Cambridge University Press, 11 June 2021. doi:10.1180/ clm.2021.15. 